\title{
p53 controls colorectal cancer cell invasion by inhibiting the NF-kB-mediated activation of Fascin
}

\author{
Xinbing Sui ${ }^{1,2, *}$, Jing Zhu ${ }^{2,3, *}$, Haimei Tang ${ }^{2}$, Chan Wang ${ }^{2}$, Jichun Zhou ${ }^{4}$, \\ Weidong Han ${ }^{1,2}$, Xian Wang ${ }^{1,2}$, Yong Fang ${ }^{1}$, Yinghua $\mathrm{Xu}^{1}{ }^{1}, \mathrm{Da} \mathrm{Li}^{1}$, Rui Chen ${ }^{2}$, \\ Junhong $\mathrm{Ma}^{6}$, Zhao Jing ${ }^{2}$, Xidong Gu${ }^{5}$, Hongming Pan ${ }^{1,2}$, Chao He ${ }^{2,3}$ \\ ${ }^{1}$ Department of Medical Oncology, Sir Run Run Shaw Hospital, Zhejiang University, Hangzhou, Zhejiang, China \\ ${ }^{2}$ Biomedical Research Center and Key Laboratory of Biotherapy of Zhejiang Province, Zhejiang University, Hangzhou, \\ Zhejiang, China \\ ${ }^{3}$ Department of Colorectal Surgery, Sir Run Run Shaw Hospital, Zhejiang University, Hangzhou, Zhejiang, China \\ ${ }^{4}$ Department of Surgical Oncology, Sir Run Run Shaw Hospital, Zhejiang University, Hangzhou, Zhejiang, China \\ ${ }^{5}$ Department of Breast Surgery, the First Affiliated Hospital of Zhejiang Chinese Medical University, Hangzhou, China \\ ${ }^{6}$ Department of Gastrointestinal Surgery, Nankai Hospital, Nankai District, Tianjin, China \\ *These authors have contributed equally to this work
}

Correspondence to:

Hongming Pan, e-mail: drpanhm@aliyun.com

Chao He, e-mail: hechao@163.com

Xinbing Sui, e-mail: hzzju@zju.edu.cn

Keywords: p53, cancer, cell invasion, NF-KB, Fascin

Received: June 05, $2015 \quad$ Accepted: August 15, $2015 \quad$ Published: August 29, 2015

\section{ABSTRACT}

p53 mutation is known to contribute to cancer progression. Fascin is an actin-bundling protein and has been recently identified to promote cancer cell migration and invasion through its role in formation of cellular protrusions such as filopodia and invadopodia. However, the relationship between p53 and Fascin is not understood. Here, we have found a new link between them. In colorectal adenocarcinomas, p53 mutation correlated with high NF-KB, Fascin and low E-cadherin expression. Moreover, this expression profile was shown to contribute to poor overall survival in patients with colorectal cancer. Wild-type p53 could inhibit NF-KB activity that repressed the expression of Fascin and cancer cell invasiveness. In contrast, in p53-deficient primary cultured cells, NF-kB activity was enhanced and then activation of NF-KB increased the expression of Fascin. In further analysis, we showed that NF-kB was a key determinant for p53 deletion-stimulated Fascin expression. Inhibition of NF-kB / p65 expression by pharmacological compound or p65 siRNA suppressed Fascin activity in p53-deficient cells. Moreover, restoration of p53 expression decreased the activation of Fascin through suppression of the NF-KB pathway. Taken together, these data suggest that a negative-feedback loop exists, whereby p53 can suppress colorectal cancer cell invasion by inhibiting the NF-KB-mediated activation of Fascin.

\section{INTRODUCTION}

Tumor metastasis, the most common cause of death for the cancer patients, is a multi-step process by which cancer cells disseminate from their primary site and subsequently form secondary distant tumor sites, where they arrest in the bloodstream and reinitiate tumor growth $[1,2]$. Although plenty of progresses have been made for the cancer treatment in the past few years, tumor metastasis is still a leading cause of cancer-related mortality.

Epithelial-mesenchymal transition (EMT) is a process, which stimulates epithelial cells to acquire the highly invasive and metastatic properties of mesenchymal 
cells [3], thus has been demonstrated to play a critical role in promoting cancer metastasis, especially for epithelium-derived carcinoma [4]. A large number of transcription factors are involved in this process. The characteristics of EMT are loss of the epithelial junction molecule E-cadherin and gain of mesenchymal markers such as Snail, Slug, Vimentin, Twist, Zeb, and Sip1 $[5,6]$. Among these transcription factors, the high expression of E-cadherin suppresses tumor invasion and metastasis, whereas the downregulation of E-cadherin facilitates malignant transformation and metastatic progression $[7,8]$.

Fascin is an actin-binding protein that expressed in a large number of human carcinomas and is usually upregulated as an important component of cancer cell epithelial to mesenchymal progression $[9,10]$. In normal epithelia, Fascin is usually present at low level or absent, however, its expression is often increased in epithelial neoplasms such as esophageal carcinomas, colorectal adenocarcinomas and other types of cancer [11-13]. Fascin can stabilize actin bundles in invasive foot structures termed invadopodia, which may confer increased metastatic potential in cancer cells [14-16]. Fascin is regulated by slug along with EMT and promotes intercalation of filopodia into mesothelial cell layers and cell invasion [17]. Recently, three groups found that $\mathrm{NF}-\mathrm{\kappa B}$ is required for the expression of Fascin in metastatic cancer cells [18-20], indicating that NF- $\mathrm{KB}$ may mediate a metastatic phenotype by specifically regulating Fascin.

The tumor suppressor p53 is widely known for its potential to induce cell death or cell cycle arrest and thereby prevent neoplastic progression $[21,22]$. Deficiency or mutation of p53 commonly occurs in approximately half of all human cancers and contributes to tumor progression $[23,24]$. Recently, p53 is shown to be associated with the process of EMT. p53 can regulate EMT and stemness or differentiation plasticity through activation of the p53-miR-200c pathway [25]. p53 is also demonstrated to control cancer cell invasion by inducing the MDM2-mediated degradation of Slug [26]. Consistent with the view that Slug can promote cancer cell invasion, Fascin is also shown to facilitate cell migration and invasion in vitro [15]. However, the role of p53 in regulating Fascin is not understood.

Here we report a new mechanism by which p53 regulates cancer invasion. It was demonstrated that wild-type p53 was able to inhibit cancer cell invasion by suppressing NF- $\kappa \mathrm{B}-$ mediated activation of Fascin, whereas, p53 deletion triggered NF- $\mathrm{KB}$-mediated activation of Fascin, thereby augmenting cancer cell invasion and metastasis. Furthermore, we showed that NF- $\mathrm{KB}$ was a key determinant for $\mathrm{p} 53$ deletionmediated the up-regulation of Fascin.

\section{RESULTS}

\section{Mutant p53 and Fascin expression correlate with poor survival time and distant metastasis of the patients with colorectal adenocarcinomas}

It has been acknowledged that mutant p53 can gain functions that accelerate malignant progression and increase cancer invasiveness and metastasis $[27,28]$. To evaluate the role of mutant p53 and Fascin in colorectal cancer progression, we collected a cohort of 75 colorectal adenocarcinoma patients and determined the expression of p53 protein and Fascin by immunohistochemistry, as well as the $\mathrm{p} 53$ mutation status by direct DNA sequencing (Fig. 1a). The correlations between clinicopathological signatures of the patients with colorectal adenocarcinomas and p53 expression are shown in Table 1. Among these patients, 53 samples $(70.7 \%)$ harbored mutant $\mathrm{p} 53$ protein expression which correlated with more distant metastasis (Table 1) and poor overall survival of patients with colorectal adenocarcinomas (Fig. 1b). Fascin was highly expressed in 49.3\% (37/75) of colorectal cancer tissues and its expression was remarkably correlated with high tumor stage (Table 1) and poor overall survival of the patients with colorectal adenocarcinomas (Fig. 1c). Taken together, these results show that mutant p53 and Fascin are associated with increased risk of metastasis and mortality in colorectal adenocarcinomas.

\section{Mutant p53 is associated with high expression of Fascin and low expression of E-cadherin in colorectal adenocarcinoma samples}

To determine the relationship between mutant $\mathrm{p} 53$ and cancer-invasion-related genes, we further investigated the expression of Fascin and E-cadherin in colorectal adenocarcinoma specimens by immunohistochemistry (Fig. 1a). As a result, most tumors with mutant p53 were associated with low expression of E-cadherin (53/53, $100 \%)$ and high expression of Fascin (32/53, 60.4\%). By contrast, the tumors with wild-type p53 were associated with high expression of E-cadherin and low expression of Fascin (Table 2), indicating that mutant p53 may induce a gain-of-function metastatic phenotype by regulating Fascin and E-cadherin.

\section{p53 and Fascin correlate with colorectal cancer cell invasion and migration in vitro}

To evaluate the effect of p53 and Fascin on cell migration, we first examined the actin dynamics by rhodamine-conjugated phalloidin staining. It has been acknowledged that increased the filopodia formation enhances cancer cell invasion [29]. Intriguingly, we found that filopodia were significantly more frequent, 
$\mathbf{a}$
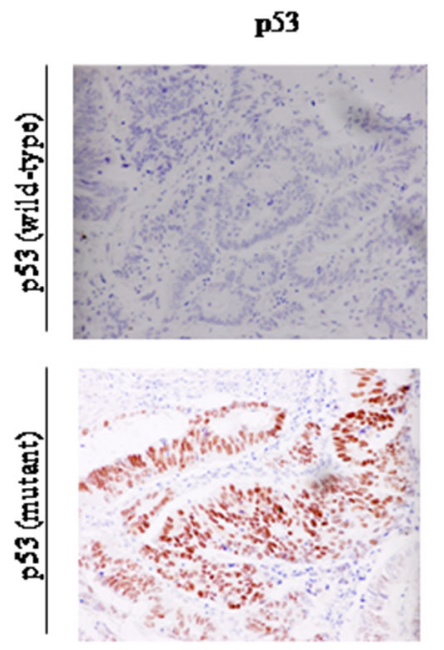

b

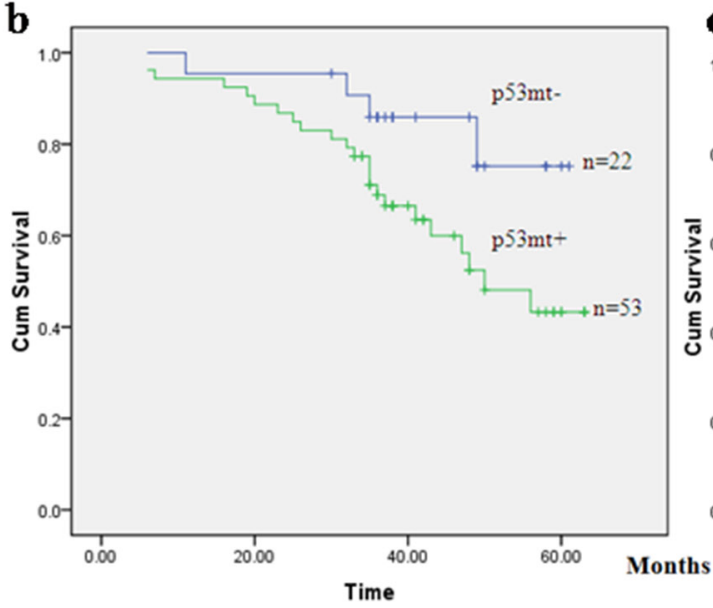

E-Cadherin
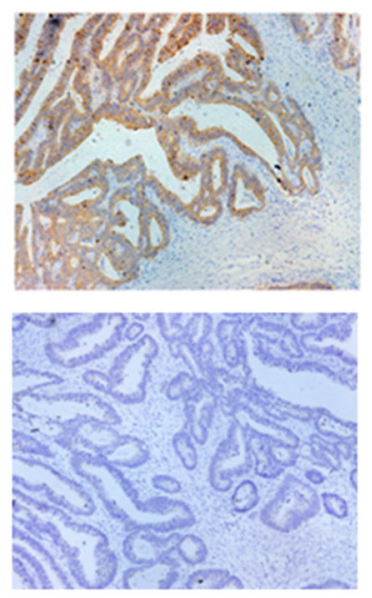

$\mathbf{c}$
Fascin
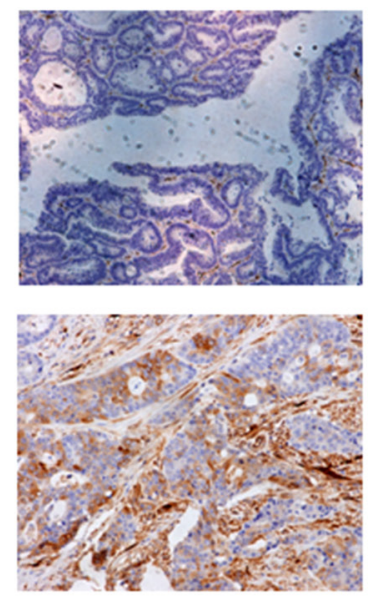

NF+kB
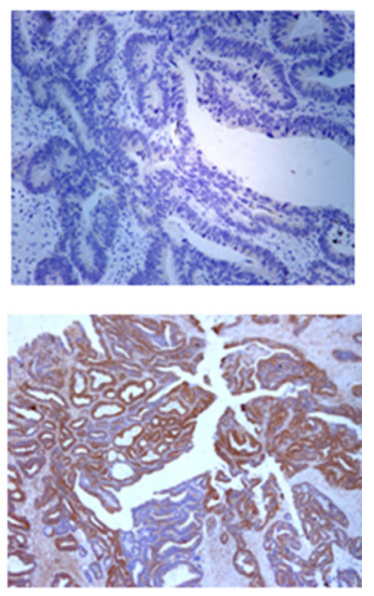

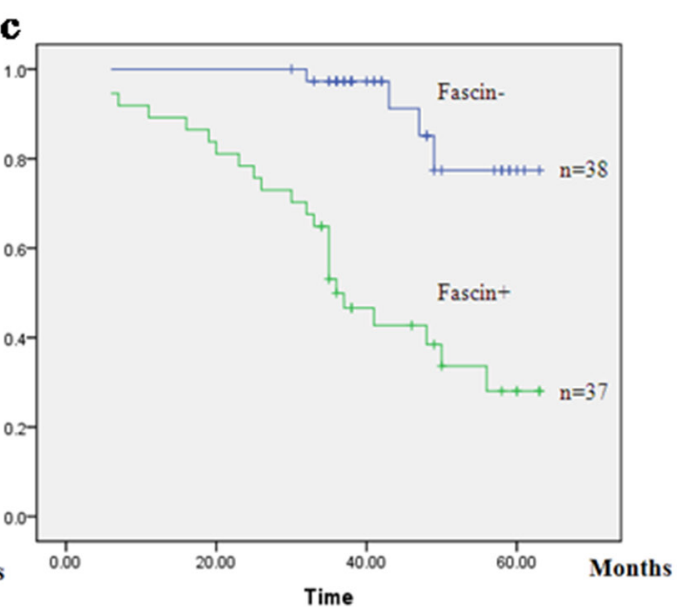

Figure 1: p53 mutation and Fascin expression are associated with poor clinical outcome in patients with colorectal adenocarcinomas. a. Immunohistochemistry of E-cadherin, Fascin and NF- $\mathrm{KB}$ in serial sections of colorectal adenocarcinoma specimens with wildtype or mutant $\mathrm{p} 53$. The original magnification: $\times 200$. b. Kaplan-Meier plots of overall survival for patients with colorectal adenocarcinomas with $\left(\mathrm{p} 53 \mathrm{mt}^{+}\right)$or without $\left(\mathrm{p} 53 \mathrm{mt}^{-}\right)$p53 mutation. c. Kaplan-Meier plots of overall survival for patients with colorectal adenocarcinomas with $\left(\mathrm{Fascin}^{+}\right)$or without $\left(\mathrm{Fascin}^{-}\right)$Fascin expression.

longer in p53-deficient cells (HCT116 p53 $3^{-/-}$and HepG2) than wtp53 cells (HCT116 p53 $3^{+/+}$and Hep3B) (Fig. 2a), indicating p53 deletion promoted cancer cell invasion and migration. Next, immunofluorescence analysis was performed to examine the expression of E-cadherin and Fascin in HCT116 p53 $3^{--}$cells. The result revealed that wtp53 increased the expression of E-cadherin (Fig. 2b) and reduced the Fascin expression (Fig. 2c). In further confirmation of the negative effect of wtp53 on cell invasive migration, transwell assay was performed. As shown in Fig. 2d, p53 expression significantly suppressed colorectal cancer cell migration after $24 \mathrm{~h}$ incubation. Moreover, Fascin knockdown using siRNA in $\mathrm{p} 53^{-/-}$cells could rescue their morphology and migratory potential. These results indicate that wtp53 could suppress cell invasion and migration via inhibiting Fascin expression.

\section{Wild-type p53 inhibits Fascin protein expression}

Thus far, little is known about transcriptional regulation of Fascin. To examine whether Fascin expression is suppressed by $\mathrm{p} 53$ in response to stimuli, we subjected HCT116 ${\mathrm{p} 53^{+/+}}^{+/}$treatment with glucose deprivation. At $12 \mathrm{~h}$ after the deprivation of glucose, Fascin protein levels were significantly downregulated in parallel with upregulated wtp53 accumulation (Fig. 3a), but Fascin mRNA levels did not change (Fig. 3b), suggesting that Fascin is not transcriptionally regulated by wtp53. By contrast, the effects of glucose deprivation on Fascin levels were abolished in $\mathrm{p} 53^{--}$mouse embryo fibroblasts (MEFs) (Fig. 3c), indicating that Fascin downregulation is through wtp53. For further confirmation, we used short interfering RNA (RNAi) to knock down p53 expression in HCT116 
Table 1: Correlation between clinicopathological background and p53 mutation status as well as NF-кB, Fascin, and E-cadherin expression in tumor specimens from 75 colorectal adenocarcinoma patients

\begin{tabular}{|c|c|c|c|c|c|c|c|c|c|c|c|c|c|c|}
\hline \multirow[b]{2}{*}{ Characteristic } & & \multirow[t]{2}{*}{$\begin{array}{l}\text { Patient } \\
\text { No. }\end{array}$} & \multicolumn{2}{|c|}{ p53 mutation } & \multirow{2}{*}{$\begin{array}{c}P \text { - } \\
\text { value }^{\dagger}\end{array}$} & \multicolumn{2}{|c|}{$\begin{array}{c}\mathrm{NF}-\kappa \mathrm{B} \\
\text { expression }\end{array}$} & \multirow{2}{*}{$\begin{array}{c}P- \\
\text { value }^{\dagger}\end{array}$} & \multicolumn{2}{|c|}{$\begin{array}{c}\text { Fascin } \\
\text { expression }\end{array}$} & \multirow{2}{*}{$\begin{array}{c}P \text { - } \\
\text { value }^{\dagger}\end{array}$} & \multicolumn{2}{|c|}{$\begin{array}{l}\text { E-cadherin } \\
\text { expression }\end{array}$} & \multirow{2}{*}{$\begin{array}{c}P- \\
\text { value }\end{array}$} \\
\hline & & & Negative & Positive & & Low & High & & Low & High & & Low & High & \\
\hline Total No. & & 75 & 22 & 53 & & 47 & 28 & & 38 & 37 & & 60 & 15 & \\
\hline \multirow[t]{2}{*}{ Gender } & Male & 42 & 12 & 30 & \multirow[b]{2}{*}{0.870} & 24 & 18 & \multirow[b]{2}{*}{0.265} & 21 & 21 & \multirow[b]{2}{*}{0.896} & 34 & 8 & \multirow[b]{2}{*}{0.816} \\
\hline & Female & 33 & 10 & 23 & & 23 & 10 & & 17 & 16 & & 26 & 7 & \\
\hline \multirow[t]{2}{*}{ Age } & $<60$ & 37 & 8 & 29 & \multirow[b]{2}{*}{0.148} & 22 & 15 & \multirow[b]{2}{*}{0.571} & 17 & 20 & \multirow[b]{2}{*}{0.420} & 31 & 6 & \multirow[b]{2}{*}{0.419} \\
\hline & $\geq 60$ & 38 & 14 & 24 & & 25 & 13 & & 21 & 17 & & 29 & 9 & \\
\hline \multirow[t]{2}{*}{ pT categories } & pT1-2 & 18 & 6 & 12 & \multirow[b]{2}{*}{0.669} & 14 & 4 & \multirow[b]{2}{*}{0.128} & 15 & 3 & \multirow[b]{2}{*}{0.001} & 12 & 6 & \multirow[b]{2}{*}{0.105} \\
\hline & pT3-4 & 57 & 16 & 41 & & 33 & 24 & & 23 & 34 & & 48 & 9 & \\
\hline \multirow[t]{2}{*}{$\mathrm{pN}$ categories } & pN0 & 32 & 12 & 20 & \multirow[b]{2}{*}{0.180} & 21 & 11 & \multirow[b]{2}{*}{0.648} & 22 & 10 & \multirow[b]{2}{*}{0.007} & 22 & 10 & \multirow[b]{2}{*}{0.036} \\
\hline & $\mathrm{pN} 1 / 2$ & 43 & 10 & 33 & & 26 & 17 & & 16 & 27 & & 38 & 5 & \\
\hline \multirow[t]{2}{*}{ pM categories } & pM0 & 66 & 22 & 44 & \multirow[b]{2}{*}{0.039} & 42 & 24 & \multirow[b]{2}{*}{0.003} & 38 & 28 & \multirow[b]{2}{*}{0.001} & 51 & 15 & \\
\hline & pM1 & 9 & 0 & 9 & & 1 & 8 & & 0 & 9 & & 9 & 0 & 0.110 \\
\hline Stage-Dukes & B & 29 & 12 & 17 & & 20 & 9 & & 23 & 6 & & 19 & 10 & \\
\hline & $\mathrm{C}$ & 37 & 10 & 27 & & 22 & 15 & & 15 & 22 & & 32 & 5 & \\
\hline & D & 9 & 0 & 9 & 0.053 & 1 & 8 & 0.009 & 0 & 9 & $<0.001$ & 9 & 0 & 0.030 \\
\hline
\end{tabular}

*High expression, more than 50\%; low expression, $50 \%$ or less.

${ }^{\dagger}$ Pearson Chi-Square test.

Table 2: Relationship between $\mathbf{p 3}$ mutation status and the protein expression of p53, NF- $\mathrm{kB}$, Fascin, and E-cadherin in tumor specimens from 75 colorectal adenocarcinoma patients

\begin{tabular}{|c|c|c|c|c|}
\hline & \multirow[b]{2}{*}{ Protein expression } & \multicolumn{2}{|c|}{ p53 mutation } & \multirow[b]{2}{*}{$P$-value } \\
\hline & & Positive $(n=53)$ & Negative $(n=22)$ & \\
\hline \multirow{2}{*}{ p53 } & High & $31(58.5 \%)$ & $6(27.3 \%)$ & \multirow[b]{2}{*}{$<0.05$} \\
\hline & Low & $22(41.5 \%)$ & $16(72.7 \%)$ & \\
\hline \multirow[t]{2}{*}{ NF-кB } & High & $24(45.3 \%)$ & $4(18.2 \%)$ & \multirow[b]{2}{*}{$<0.05$} \\
\hline & Low & $29(54.7 \%)$ & $18(81.8 \%)$ & \\
\hline \multirow[t]{2}{*}{ Fascin } & High & $32(60.4 \%)$ & $5(22.7 \%)$ & \multirow[b]{2}{*}{$<0.05$} \\
\hline & Low & $21(39.6 \%)$ & $17(77.3 \%)$ & \\
\hline \multirow[t]{2}{*}{ E-cadherin } & High & $0(0 \%)$ & $15(68.2 \%)$ & \multirow[b]{2}{*}{$<0.05$} \\
\hline & Low & $53(100 \%)$ & $7(31.8 \%)$ & \\
\hline
\end{tabular}

*High expression, more than $50 \%$; low expression, $50 \%$ or less.

†Pearson Chi-Square test. 


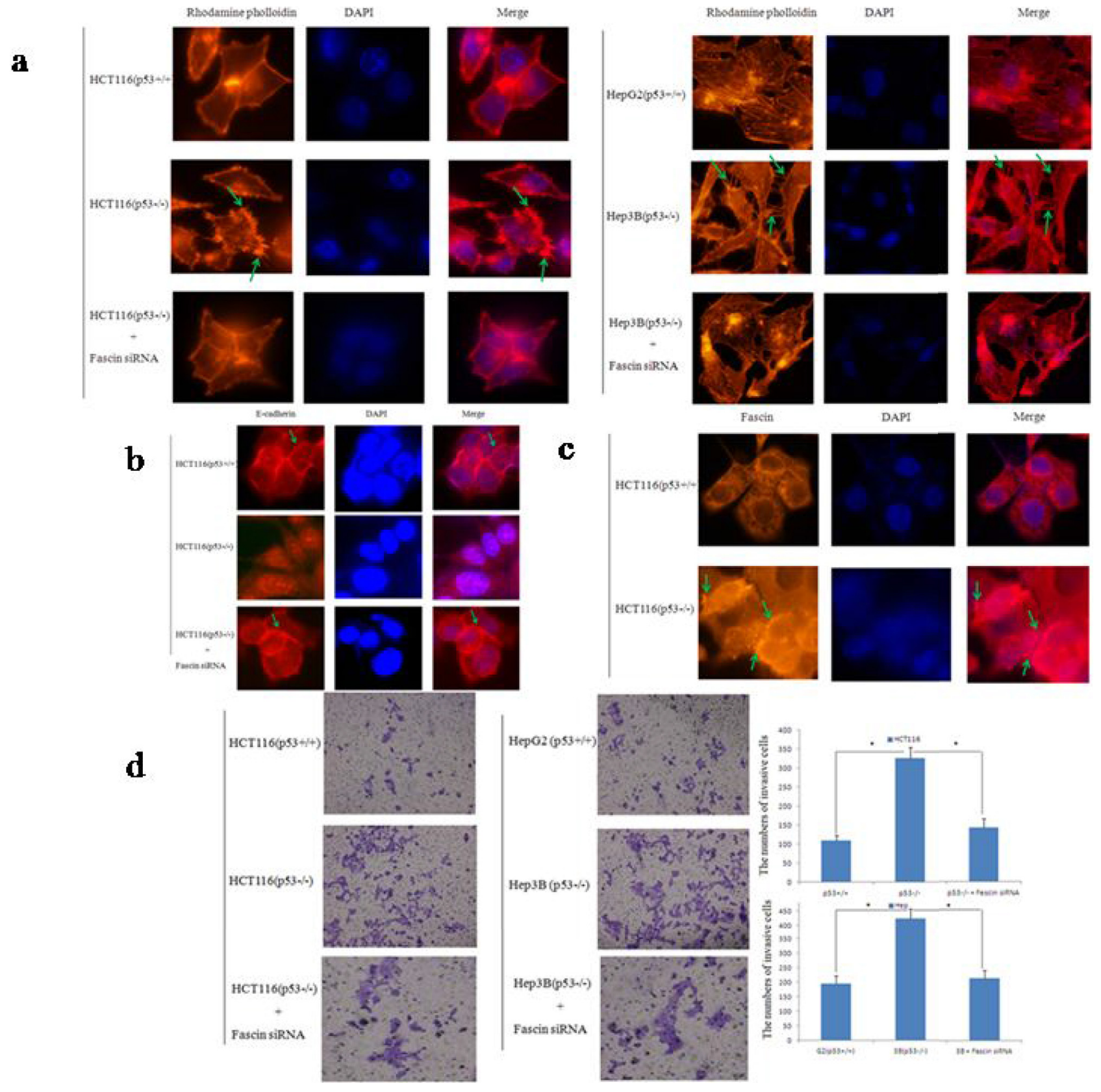

Figure 2: p53 and Fascin correlate with colorectal cancer cell migration in vitro. a. Cell morphology and cytoskeleton analysis by immunofluorescence microscopy. Green stars indicate filopodia. The experiments were performed in triplicate. b. Representative images of E-cadherin expression in HCT116 cells. Photos were taken under $\times 400$ magnification. Green stars indicate Fascin wxpression. The experiments were performed in triplicate. c. Representative images of Fascin expression in HCT116 cells. Photos were taken under $\times 400$ magnification. Green stars indicate Fascin wxpression. The experiments were performed in triplicate. d. Transwell invasion assay by the 24-transwell system and quantitative analysis. The pictures were taken $24 \mathrm{~h}$ after seeding (original magnification: $\times 100$ ). The numbers of invasive cells were counted in five representative high power fields per transwell. Three independent experiments were carried out in triplicate.

p53 $3^{+/+}$cells and transfected HCT116 $\mathrm{p} 53^{-/-}$cells by $\mathrm{p} 53$ adenovirus plasmid. As shown in Fig. 3d, Knockdown of wtp53 increased Fascin expression, conversely, restoration of wtp53 caused a decrease in Fascin protein levels. These data suggest that wtp53 suppressed Fascin protein expression.

\section{p53 deletion stimulates Fascin expression via the NF- $\kappa \mathrm{B}$ signaling pathway}

NF- $\mathrm{kB}$ has been demonstrated to contribute to metastatic phenotypes by specifically regulating Fascin $[30,31]$. To investigate whether canonical NF- $\kappa B$ signal 
$\mathbf{a}$

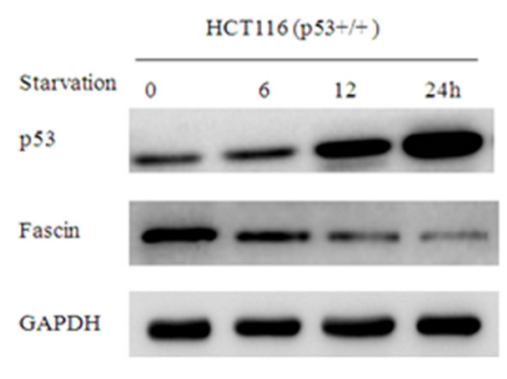

c

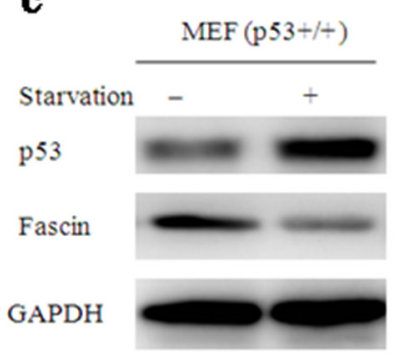

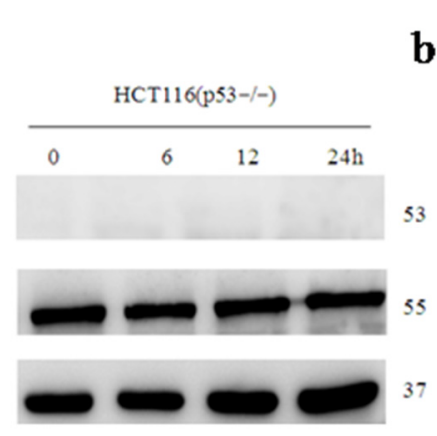

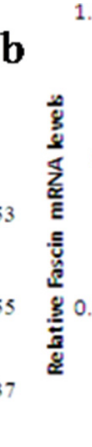

.57.

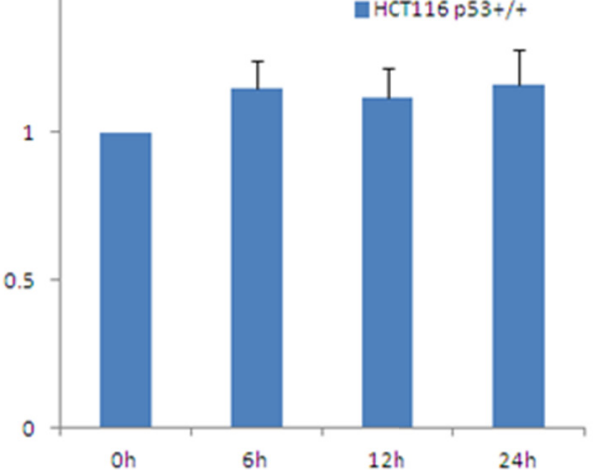

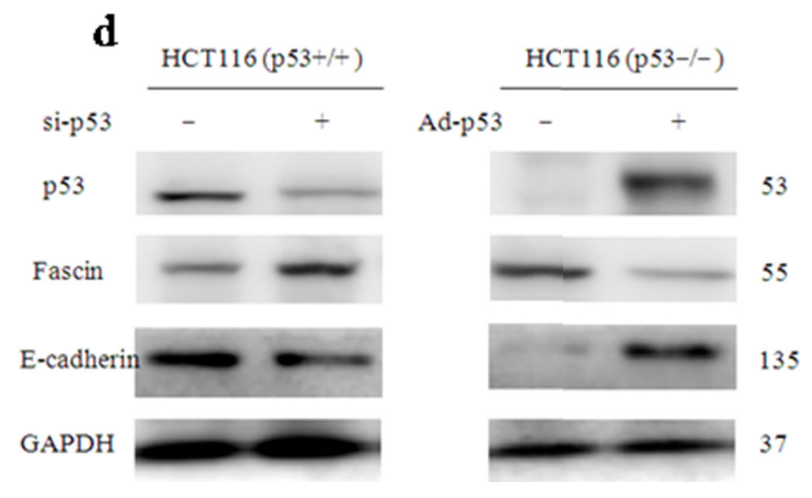

Figure 3: Wild-type p53 inhibits Fascin protein expression. a-c. Glucose deprivation-induced p53 activation decreases the level of Fascin protein but not that of Fascin mRNA (the blots were cropped, and the full-length blots are included in the supplementary information). d. The effect of p53 on Fascin expression when the cells were transfected with p53-siRNA or p53 adenovirus plasmid.

is a key determinant for p53 deletion-stimulated the Fascin expression, we first assessed the activation of two typical

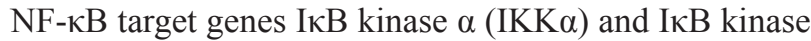
$\beta$ (IKK $\beta$ ) by RT-PCR and western blotting. We found that the activity of IKK $\alpha / \beta$ was remarkably increased in $\mathrm{p} 53^{-/-}$ cells, compared with wtp53 cells (Fig. 4a), suggesting that the upstream of NF- $\mathrm{kB}$ signal was activated in $\mathrm{p} 53^{-/-}$cells. Next, the abundance of NF- $\mathrm{kB}$ was examined by western blotting (Fig. 4b). The result confirmed that NF- $\kappa B$ was significantly activated in $\mathrm{p} 53^{-/-}$cells, accompany with the down-regulation of epithelial marker E-cadherin and the up-regulation of mesenchymal markers $\mathrm{N}$-cadherin, Vimentin and Fascin, indicating that p53 deletion activated $\mathrm{NF}-\mathrm{\kappa B}$ signaling pathway and triggered EMT transition which facilitated cancer cell invasion and migration. In the further confirmation, $\mathrm{p} 53^{-/-}$cells were transfected with a p53 adenovirus for $48 \mathrm{~h}$. As a result, exogenous expression of wtp53 suppressed NF- $\mathrm{kB}$ activation and decreased Fascin level in p53 $3^{--}$cells (Fig. 4c).

To determine whether Fascin expression is regulated by $\mathrm{p} 53$ via $\mathrm{NF}-\mathrm{\kappa B}$ signaling pathway, we specifically attenuated NF- $\mathrm{\kappa B}$ activation in $\mathrm{p} 53^{-/-}$MEFs using IMD-0354 $(10 \mu \mathrm{M})$, a selective inhibitor of IKK- 2 that is necessary for induction of NF- $\mathrm{KB}$ p 65 nuclear translocation [32]. The inhibition of NF-kB by IMD-0354 suppressed Fascin expression in p53 $3^{-/}$MEFs (Fig. 4d).
We also performed p65 knockdown using siRNA in p53-/MEFs and obtained similar results (Fig. 4d). Moreover, exogenous expression of wtp53 decreased Fascin levels in $\mathrm{p} 53^{-/-}$MEFs but not in $\mathrm{p} 53^{-/-}$p $65^{-/-}$MEFs (Fig. 4e), indicating that wtp53 may act inhibit Fascin activation through the suppression of NF-kB signal.

\section{The signature of p53 mutation, high NF- $\mathrm{B}$ and Fascin, and low E-cadherin expression correlates with poor overall survival and early metastasis in patients with colorectal adenocarcinomas}

As shown in Table 2, the patients with p53 mutation showed high NF- $\kappa \mathrm{B}$ and Fascin expression and low E-cadherin expression in colorectal adenocarcinomas, whereas wt p53 was correlated with low NF-kB and Fascin expression and high E-cadherin expression. To determine whether p53 mutation is related with the expression profiles of NF- $\mathrm{KB}$ and Fascin, we analyzed the prognostic significances of p53 mutation status, NF- $\mathrm{kB}$ and Fascin expression in 75 patients with colorectal adenocarcinomas by Kaplan-Meier analysis and log-rank test. As a result, the patients with a p $53 \mathrm{mt}^{+}-\mathrm{NF}-\mathrm{KB}^{+}-\mathrm{Fascin}^{+}$expression profile had a significantly poorer overall survival than the p53mt ${ }^{-}-\mathrm{NF}_{-} \mathrm{kB}^{-}-$Fascin $^{-}$group (Fig. 5a). Thus, our results indicate that p53 mutation and high expression of 

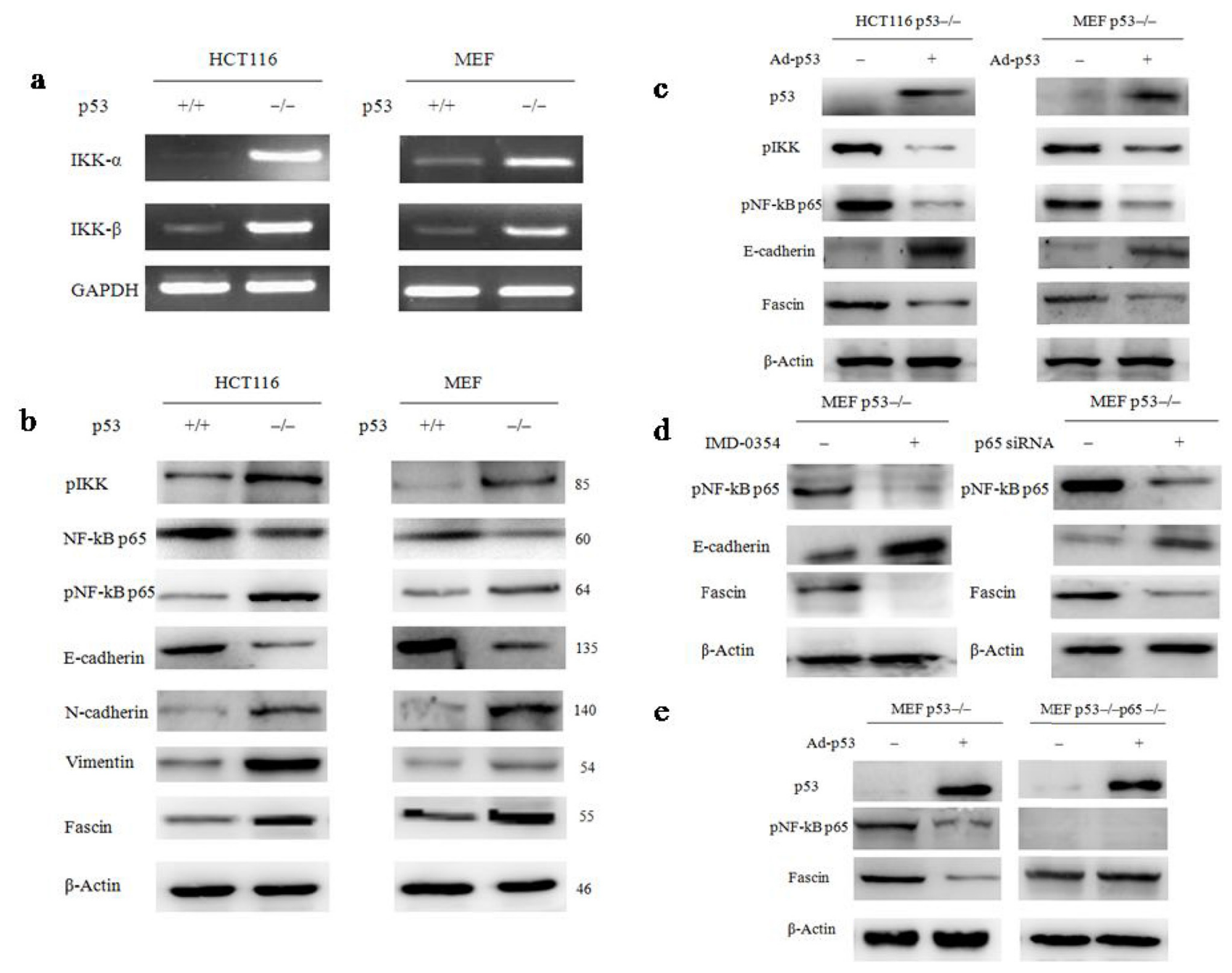

Figure 4: NF-кB is a key determinant for p53 deletion-mediated the up-regulation of Fascin. a. The expression of IKK $\alpha$ and IKK $\beta$, typical NF- $\kappa$ B target genes, in wild-type and $\mathrm{p} 53^{-/-}$HCT116s and MEFs was examined by RT-PCR. b. The expression of several key NF- $\kappa$ B signals regulators, E-Cadherin, N- Cadherin, Vimentin and Fascin were examined by western blotting (the blots were cropped, and the full-length blots are included in the supplementary information). c. The expression of pIKK, pNF- $\mathrm{kB}$ p65, E-Cadherin and Fascin in $\mathrm{p} 53^{-/-}$cells after the cells were transfected with $\mathrm{p} 53$ adenovirus plasmid. $\mathbf{d}$. The expression of pNF- $\mathrm{kB}$ p 65 and Fascin in p53 ${ }^{-/-} \mathrm{MEFs}$ after the cells were treated with the compound IMD-0354 or p65 siRNA. e. p53 deletion-induced Fascin activation is NF- $\mathrm{kB}-\mathrm{dependent.}$ $\mathrm{p} 53^{-/-}$and $\mathrm{p} 53^{-/-} \mathrm{p} 65^{-/-}$MEFs were infected with a p53 adenovirus plasmid for $48 \mathrm{~h}$, and cell lysates were analysed by western blotting with the indicated antibodies (the blots were cropped, and the full-length blots are included in the supplementary information).

NF- $\mathrm{kB}$ and Fascin hold potential as prognostic markers in colorectal adenocarcinomas.

\section{DISCUSSION}

It is increasingly appreciated that tumor suppressor gene $\mathrm{p} 53$ plays a functional role in tumor initiation and progression, however, the function of p53 in tumor metastasis and EMT has not been well elaborated. In this study, we show a novel mechanism by which p53 inhibits colorectal cancer cell invasion through negative regulation of Fascin, a metastatic phenotype marker. We report that p53 mutation is correlated with low E-cadherin expression and high Fascin expression, as well as with poor overall survival. Moreover, p53 deletion promotes epithelial marker E-cadherin expression and decreases mesenchymal marker Fascin level, indicating p53 deletion triggers EMT transition and enhances colorectal cancer cell invasion and migration. In agreement with above results, the transwell assay also shows that Loss of $\mathrm{p} 53$ promotes the invasion and metastasis ability of colorectal cancer cells.

Emerging evidence has suggested that NF- $\mathrm{KB}$ pathway plays an important role in cell proliferation, motility, apoptosis, metabolism and DNA repair [33, 34]. Recently, several researches indicate NF- $\mathrm{KB}$ signals play an important role in the regulation of Fascin, however, whether Fascin expression is regulated by $\mathrm{p} 53$ via NF- $\mathrm{\kappa B}$ signaling pathway remains unresolved. In the present 

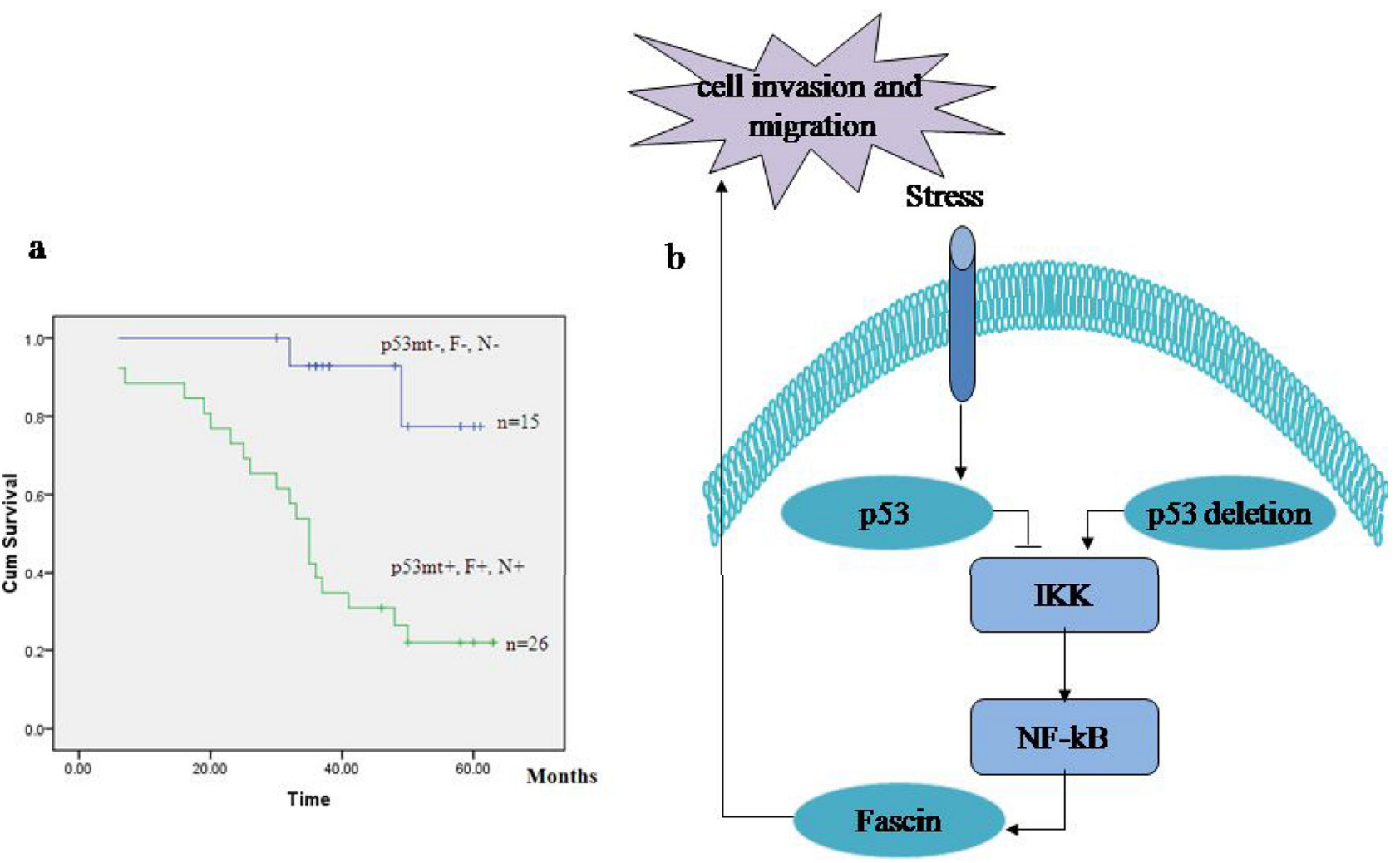

Figure 5: The signature of p53 mutation, high NF-кB and Fascin and low E-cadherin expression in patients with colorectal adenocarcinomas. a. Kaplan-Meier analysis of overall survival for patients with colorectal adenocarcinomas with $\mathrm{p} 53 \mathrm{mt}{ }^{+}-$ $\mathrm{NF}-\kappa^{+} \mathrm{B}^{+}$Fascin $^{+}$versus p $53 \mathrm{mt}^{-}-\mathrm{NF}-\kappa^{-}-$Fascin $^{-}$. b. Model of the proposed link between $\mathrm{p} 53$, NF- $\mathrm{BB}$, Fascin and cell invasion.

study, we demonstrate that p53 deletion stimulates NF- $\mathrm{BB}$ activation and high Fascin level in cancer cells. Exogenous expression of wtp53 inhibits this effect. In further analysis, we show that NF- $\kappa \mathrm{B}$ activation is a key determinant in p53 deletion-activated Fascin expression. The inhibition of NF- $\kappa$ B by the compound IMD-0354 or p65 siRNA suppresses Fascin expression in p53 ${ }^{-/-}$MEFs, moreover, exogenous expression of wtp53 decreases Fascin levels in $\mathrm{p} 53^{-/-}$MEFs but not in $\mathrm{p} 53^{-/} \mathrm{p} 65^{-/-}$MEFs. Taken together, our results demonstrate that $\mathrm{p} 53$ can suppress colorectal

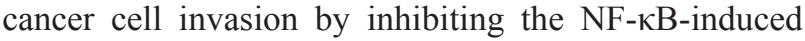
activation of Fascin.

In conclusion, our results provide a new negativefeedback molecular mechanism by which loss of p53 facilitates cell invasion and migration via NF- $\mathrm{B}$-mediated activation of Fascin (Fig. 5b). This finding may offer new prognostic markers for the patients with colorectal adenocarcinomas.

\section{MATERIALS AND METHODS}

\section{Cell lines, reagents and tumor samples}

The human colorectal carcinoma HCT116 p53 $3^{+/+}$, HCT116 $553^{-/}$cell lines were kindly given by Mian Wu.

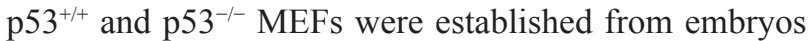

of the corresponding mice generated by crossing p53 heterozygote mice (B6.129S2-Trp53tm1Tyj/J, The Jackson Laboratory, Bar Harbor, ME). HepG2 and Hep3B cells were purchased from ATCC (LGC Standards SLU, Barcelona, Spain). The cell lines were maintained in McCoy's 5A or Dulbecco's modified Eagle's medium (DMEM; Gibco BRL, Rockville, MD, USA) with 10\% fetal bovine serum (FBS), 100 units/mL penicillin, $100 \mu \mathrm{g} /$ $\mathrm{mL}$ streptomycin (Invitrogen), and $2 \mathrm{mmol} / \mathrm{L}$ L-glutamine at $37^{\circ} \mathrm{C}$ in a humidified atmosphere of $95 \%$ air and $5 \%$ $\mathrm{CO}_{2}$. The IKK-2 inhibitor IMD-0354 was purchased from Sigma Aldrich, St Louis, MO.

A total of 75 colorectal adenocarcinoma patients who underwent surgery between February 2004 and June 2006 at the Sir Run Run Shaw Hospital (Hangzhou, Zhejiang, China) were investigated. Patients who received pre-operative chemotherapy were excluded. This study was approved and monitored by the ethics committee of Sir Run Run Shaw Hospital, Zhejiang University.

\section{RNA extraction and RT-PCR}

RNA was isolated from the cancer cells using a Qiagen RNAeasy kit (QIAGEN, Tokyo, Japan). Briefly, $1 \mu \mathrm{g}$ of RNA was used to synthesize the first-strand cDNA using the Superscript system (Life Technologies, 
Inc.) in accordance with the manufacturer's instructions. RT-PCR reactions for IKK $\alpha$ and IKK $\beta$ were performed. For sequencing, the following primers were used: p53 Exon 5-8 forward 5'-TCTTCCTACAGTACTCCCCT-3', reverse 5'-GCTTGCTTACCTCGCTTAGT-3'; p53 Exon 7-8 forward 5'-TAGGTTGGCTCTGACTGT-3', reverse 5'-GCTTGCTTACCTCGCTTAGT-3'.

\section{RNA interference}

Cells were transfected with p53 siRNA (Cell Signaling Technology, \#6231) or NF-кB p65 siRNA (Cell Signaling Technology, \#6261) via LipofectAMINE RNAi max (Invitrogen, 13778150) according to the manufacturer's instructions.

\section{Transwell invasion assay}

Cells were trypsinized and resuspended in DMEM containing $1 \%$ fetal bovine serum at a density of $1 \times 10^{6}$ cells/ml. $100 \mu \mathrm{l}$ of the cell suspension was added into the upper chamber (was coated with Matrigel) of a transwell (Corning, Corning, NY, USA) consisted of inserts containing $8-\mu \mathrm{m}$ pore-size PET membrane. DMEM (600 $\mu \mathrm{l}$ ) containing $10 \%$ fetal bovine serum was placed in the lower chamber. After a $24 \mathrm{~h}$ incubation at $37^{\circ} \mathrm{C}$ cells remained in the upper chamber was removed carefully by cotton swab and the membrane was cut off by an operating knife. The side facing lower chamber was stained with $0.05 \%$ crystal violet and attached cells were counted under a light microscope. The experiment was performed three times.

\section{Immunofluorescence}

Cells were cultured on glass coverslips and fixed in $3.7 \%$ paraformaldehyde in phosphate buffered saline ( $\mathrm{pH} 7.4$ ) for $10 \mathrm{~min}$, permeabilized in $0.1 \%$ Triton $\mathrm{X}-100$ in phosphate buffered saline for $4 \mathrm{~min}$, blocked with $1 \%$ bovine serum albumin/phosphate buffered saline for $1 \mathrm{~h}$, and then incubated at room temperature for $1 \mathrm{~h}$ with rhodamine-conjugated phalloidin (Invitrogen, Carlsbad, CA, USA) or Fascin at 1:100 in blocking solution. Nuclei were counterstained with DAPI. Images were acquired with an Olympus BX51 microscope.

\section{Antibodies}

p53 antibody (DO-1): sc-126 were obtained from Santa Cruz Biotechnology (Santa Cruz, CA, USA). NF-

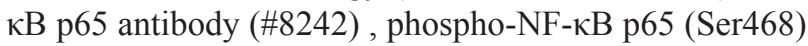
(\#3039), Phospho-IKK $\alpha \quad$ (Ser176)/IKK $\beta$ (Ser177) (C84E11) Rabbit mAb (\#2078), E-Cadherin (24E10) (\#3195), N-Cadherin (D4R1H) (\#13116) and phosphoNF- $\kappa$ B p65 (Ser 536) (\#3031) antibodies were obtained from Cell Signaling (Beverly, MA, USA). Fascin antibody (ab78599) was obtained from Abcam. The anti- $\beta$-actin antibody (AC-15) was purchased from Sigma-Aldrich (St. Louis, MO, USA).

\section{Immunohistochemistry staining}

The ChemMate EnVision Detection Kit (DAKO, Carpinteria, CA, USA) was used for immunohistochemistry according to company's recommended procedure. Briefly, after being deparaffinized and hydrated, the paraffin-embedded sections were placed in $0.01 \mathrm{M}$ sodium citratebuffe $\mathrm{r}$ ( $\mathrm{pH}$ 6.0), and subjected to pressure cooker treatment for $2 \mathrm{~min}$ at full pressure with a domestic pressure cooker. After cooling to room temperature, the slides were rinsed with Tris buffered saline $(0.05 \mathrm{M}$ Tris $/ 0.15 \mathrm{M} \mathrm{NaCl}$, $\mathrm{pH}$ 7.6). The endogenous peroxidase activity was blocked by incubating the sections with $3 \%$ hydrogen peroxide. The sections were incubated with the primary antibody overnight at $4^{\circ} \mathrm{C}$. Then, the ChemMate EnVision/HRP, Rabbit/Mouse (ENV) reagent was applied to the sections, followed by application of ChemMate DABt Chromogen included in the kit. The slides were lightly counterstained with hematoxylin.

\section{Western blot analysis}

Cells were harvested from cultured dishes and were lysed in a lysis buffer [20 mM Tris- $\mathrm{HCl} \mathrm{pH} 7.6,1 \mathrm{mM}$ EDTA, $140 \mathrm{mM} \mathrm{NaCl}, 1 \% \mathrm{NP}-40,1 \%$ aprotinin, $1 \mathrm{mM}$ phenylemethylsulfonyl fluoride (PMSF), $1 \mathrm{mM}$ sodium vanadate]. Protein concentration was determined using a BCA Protein Assay Kit (Pierce). Cell lysates (40 $\mu \mathrm{g}$ protein/line) were separated on a 5 to $20 \%$ Tris-Tricine Ready Gel SDS-PAGE (Bio-Rad) for nitrocellulose membrane blotting. The blotted membranes were blocked with $5 \%$ skim milk for $1 \mathrm{~h}$ and were incubated with primary antibodies. The immunoreactive bands were visualized by enhanced chemiluminescence using horseradish perox-idase-conjugated IgG secondary antibodies. Band density was measured by densitometry, quantified using gel plotting macros of NIH image 1.62, and normalized to an indicated sample in the identical membrane.

\section{Statistical analyses}

Results are expressed as values of mean \pm standard deviation (SD). Statistical analysis was performed using SPSS 16.0 for Windows (SPSS Inc., Chicago, IL, USA). The correlation coefficient of two factors was evaluated using Chi-square and Fisher's exact tests. The survival of patients with colorectal adenocarcinomas was compared using the Kaplan-Meier method, and differences between the survival curves were tested using the logrank test. A $P$-value less than 0.05 is considered significant. 


\section{ACKNOWLEDGMENTS}

We thank Mian Wu for providing HCT116 p53 HCT116 p53 $3^{-/}$cell lines.

This study is supported by grants from National Natural Science Foundation of China (grant No. 81301891), Zhejiang Provincial Natural Science Foundation of China (grant No. LQ13H160008), Zhejiang province science and technology project of TCM (grant No. 2015ZB033) and Zhengshu Medical Elite Scholarship Fund.

\section{Author contributions}

X.S., H.P. and C.H. designed the study. X.S. wrote the main manuscript text. X.S., J.Z., H.T., C.W., J.Z., X.W., Y.F., W.H., D.L., Y.X., Z.J., R.C., J.M. and X.G. performed experiments. J.Z. and H.T. prepared figures. J.Z. and X.G. did the statistical analyses. All authors reviewed the manuscript.

\section{CONFLICTS OF INTEREST}

The authors declare no conflict of interest.

None of the contents of this manuscript has been previously published or is under consideration elsewhere. All the authors read and approved the final version of the manuscript prior to submission.

\section{REFERENCES}

1. Steeg PS. Tumor metastasis: mechanistic insights and clinical challenges. Nature medicine. 2006; 12:895-904.

2. Mina LA, Sledge GW Jr. Rethinking the metastatic cascade as a therapeutic target. Nature reviews Clinical oncology. $2011 ; 8: 325-332$.

3. Thiery JP, Acloque H, Huang RY, Nieto MA. Epithelialmesenchymal transitions in development and disease. Cell. 2009; 139:871-890.

4. Tam WL, Weinberg RA. The epigenetics of epithelialmesenchymal plasticity in cancer. Nature medicine. 2013; 19:1438-1449.

5. Tsai JH, Yang J. Epithelial-mesenchymal plasticity in carcinoma metastasis. Genes \& development. 2013; 27:2192-2206.

6. Stewart CJ, McCluggage WG. Epithelial-mesenchymal transition in carcinomas of the female genital tract. Histopathology. 2013; 62:31-43.

7. Wang CA, Drasin D, Pham C, Jedlicka P, Zaberezhnyy V, Guney M, Li H, Nemenoff R, Costello JC, Tan AC, Ford HL. Homeoprotein Six2 promotes breast cancer metastasis via transcriptional and epigenetic control of E-cadherin expression. Cancer research. 2014; 74:7357-7370.

8. Park JW, Jang SH, Park DM, Lim NJ, Deng C, Kim DY, Green JE, Kim HK. Cooperativity of E-cadherin and
Smad4 loss to promote diffuse-type gastric adenocarcinoma and metastasis. Molecular cancer research: MCR. 2014; 12:1088-1099.

9. Teng Y, Xu S, Yue W, Ma L, Zhang L, Zhao X, Guo Y, Zhang C, Gu M, Wang Y. Serological investigation of the clinical significance of fascin in non-small-cell lung cancer. Lung cancer. 2013; 82:346-352.

10. Esnakula AK, Ricks-Santi L, Kwagyan J, Kanaan YM, DeWitty RL, Wilson LL, Gold B, Frederick WA, Naab TJ. Strong association of fascin expression with triple negative breast cancer and basal-like phenotype in African-American women. Journal of clinical pathology. 2014; 67:153-160.

11. Hashimoto $Y$, Ito $T$, Inoue $H$, Okumura $T$, Tanaka $E$, Tsunoda S, Higashiyama M, Watanabe G, Imamura M, Shimada Y. Prognostic significance of fascin overexpression in human esophageal squamous cell carcinoma. Clinical cancer research: an official journal of the American Association for Cancer Research. 2005; 11:2597-2605.

12. Hashimoto Y, Skacel M, Lavery IC, Mukherjee AL, Casey G, Adams JC. Prognostic significance of fascin expression in advanced colorectal cancer: an immunohistochemical study of colorectal adenomas and adenocarcinomas. BMC cancer. 2006; 6:241.

13. Qualtrough D, Singh K, Banu N, Paraskeva C, Pignatelli $\mathrm{M}$. The actin-bundling protein fascin is overexpressed in colorectal adenomas and promotes motility in adenoma cells in vitro. British journal of cancer. 2009; 101:1124-1129.

14. Li A, Dawson JC, Forero-Vargas M, Spence HJ, Yu X, Konig I, Anderson K, Machesky LM. The actin-bundling protein fascin stabilizes actin in invadopodia and potentiates protrusive invasion. Current biology: CB. 2010; 20:339-345.

15. Machesky LM, Li A. Fascin: Invasive filopodia promoting metastasis. Communicative \& integrative biology. 2010; 3:263-270.

16. Van Audenhove I, Boucherie C, Pieters L, Zwaenepoel O, Vanloo B, Martens E, Verbrugge C, HassanzadehGhassabeh G, Vandekerckhove J, Cornelissen M, De Ganck A, Gettemans J. Stratifying fascin and cortactin function in invadopodium formation using inhibitory nanobodies and targeted subcellular delocalization. FASEB journal: official publication of the Federation of American Societies for Experimental Biology. 2014; 28:1805-1818.

17. Li A, Morton JP, Ma Y, Karim SA, Zhou Y, Faller WJ, Woodham EF, Morris HT, Stevenson RP, Juin A, Jamieson NB, MacKay CJ, Carter CR, Leung HY, Yamashiro S, Blyth K, et al. Fascin is regulated by slug, promotes progression of pancreatic cancer in mice, and is associated with patient outcomes. Gastroenterology. 2014; 146:1386-1396 e1381-1317.

18. Kress AK, Kalmer M, Rowan AG, Grassmann R, Fleckenstein B. The tumor marker Fascin is strongly induced by the Tax oncoprotein of HTLV-1 through NF-kappaB signals. Blood. 2011; 117:3609-3612. 
19. Mohr CF, Kalmer M, Gross C, Mann MC, Sterz KR, Kieser A, Fleckenstein B, Kress AK. The tumor marker Fascin is induced by the Epstein-Barr virus-encoded oncoprotein LMP1 via NF-kappaB in lymphocytes and contributes to their invasive migration. Cell communication and signaling: CCS. 2014; 12:46.

20. Snyder M, Huang J, Huang XY, Zhang JJ. A signal transducer and activator of transcription 3.Nuclear Factor kappaB (Stat3.NFkappaB) complex is necessary for the expression of fascin in metastatic breast cancer cells in response to interleukin (IL)-6 and tumor necrosis factor (TNF)-alpha. The Journal of biological chemistry. 2014; 289:30082-30089.

21. Lane DP. Cancer. p53, guardian of the genome. Nature. 1992; 358:15-16.

22. Xue W, Zender L, Miething C, Dickins RA, Hernando E, Krizhanovsky V, Cordon-Cardo C, Lowe SW. Senescence and tumour clearance is triggered by $\mathrm{p} 53$ restoration in murine liver carcinomas. Nature. 2007; 445:656-660.

23. Soussi T, Wiman KG. Shaping genetic alterations in human cancer: the p53 mutation paradigm. Cancer cell. 2007; 12:303-312.

24. Muller PA, Vousden KH. p53 mutations in cancer. Nature cell biology. 2013; 15:2-8.

25. Chang CJ, Chao CH, Xia W, Yang JY, Xiong Y, Li CW, Yu WH, Rehman SK, Hsu JL, Lee HH, Liu M, Chen CT, Yu D, Hung MC. p53 regulates epithelial-mesenchymal transition and stem cell properties through modulating miRNAs. Nature cell biology. 2011; 13:317-323.

26. Wang SP, Wang WL, Chang YL, Wu CT, Chao YC, Kao SH, Yuan A, Lin CW, Yang SC, Chan WK, Li KC, Hong TM, Yang PC. p53 controls cancer cell invasion by inducing the MDM2-mediated degradation of Slug. Nature cell biology. 2009; 11:694-704.

27. Muller PA, Vousden KH. Mutant p53 in cancer: new functions and therapeutic opportunities. Cancer cell. 2014; 25:304-317.
28. Said R, Hong DS, Warneke CL, Lee JJ, Wheler JJ, Janku F, Naing A, Falchook GS, Fu S, Piha-Paul S, Tsimberidou AM, Kurzrock R. P53 mutations in advanced cancers: clinical characteristics, outcomes, and correlation between progression-free survival and bevacizumabcontaining therapy. Oncotarget. 2013; 4:705-714.

29. Li Y, Zhang Z, Zhou X, Li L, Liu Q, Wang Z, Bai X, Zhao Y, Shi H, Zhang X, Ye L. The oncoprotein HBXIP enhances migration of breast cancer cells through increasing filopodia formation involving MEKK2/ERK1/2/Capn4 signaling. Cancer letters. 2014; 355:288-296.

30. Grothey A, Hashizume R, Ji H, Tubb BE, Patrick CW Jr, Yu D, Mooney EE, McCrea PD. C-erbB-2/ HER-2 upregulates fascin, an actin-bundling protein associated with cell motility, in human breast cancer cell lines. Oncogene. 2000; 19:4864-4875.

31. Al-Alwan M, Olabi S, Ghebeh H, Barhoush E, Tulbah A, Al-Tweigeri T, Ajarim D, Adra C. Fascin is a key regulator of breast cancer invasion that acts via the modification of metastasis-associated molecules. PloS one. 2011; 6:e27339.

32. Norden R, Nystrom K, Olofsson S. Activation of host antiviral RNA-sensing factors necessary for herpes simplex virus type 1-activated transcription of host cell fucosyltransferase genes FUT3, FUT5, and FUT6 and subsequent expression of sLe(x) in virus-infected cells. Glycobiology. 2009; 19:776-788.

33. Tada Y, Kokabu S, Sugiyama G, Nakatomi C, Aoki K, Fukushima H, Osawa K, Sugamori Y, Ohya K, Okamoto M, Fujikawa T, Itai A, Matsuo K, Watanabe S, Jimi E. The novel IkappaB kinase beta inhibitor IMD-0560 prevents bone invasion by oral squamous cell carcinoma. Oncotarget. 2014; 5:12317-12330.

34. Kawauchi K, Araki K, Tobiume K, Tanaka N. p53 regulates glucose metabolism through an IKK-NF-kappaB pathway and inhibits cell transformation. Nature cell biology. 2008; 10:611-618. 